\title{
Desempenho de Cutias (Dasyprocta prymnolopha) Criadas em Cativeiro do Nascimento até o Desmame em Teresina, Piauí
}

\author{
João Batista Lopes ${ }^{1}$, Rildênio Renato Cavalcante ${ }^{2}$, Mônica Marcos de Almeida², Maria Acelina \\ Martins de Carvalho ${ }^{3}$, Sandovaldo Gonçalves de Moura ${ }^{2}$, Laí Alves Dantas Filho², Washington \\ Luis Ferreira Conceição ${ }^{2}$
}

\begin{abstract}
RESUMO - Objetivou-se avaliar a influência do sexo e do tipo de parto (simples ou duplo) sobre peso, comprimento corporal, perímetro torácico ao nascer e ao desmame de cutias, bem como o ganho de peso total e o ganho médio diário, em busca de subsídios que contribuam para a preservação e a exploração racional desses animais. Foram avaliados 41 animais (26 machos e 15 fêmeas), em delineamento experimental inteiramente casualizado em esquema fatorial 2x2, associando-se os tratamentos: sexo (macho, fêmea) e tipo de parto (duplo e simples). O peso médio, o perímetro torácico médio, o comprimento corporal dos filhotes ao nascer para, machos e fêmeas, foram de 147,62 g e 146,25 g; 12,26 cm e 12,17 cm; 19,91 cm e 19,39 cm, respectivamente. Para as mesmas variáveis, os valores ao desmame para machos e fêmeas foram 481,92 g e 509,33 g; 17,12 cm e 16,85 cm; $30,07 \mathrm{~cm}$ e 30,42 cm, respectivamente. Não houve diferença significativa entre os parâmetros avaliados, quando comparados ao sexo e ao tipo de parto (simples ou duplo). Concluiu-se que o peso de filhotes de cutia ao nascer e ao desmame não tem relação com o sexo e o tipo de parto; o peso médio, o comprimento corporal e o perímetro torácico de filhotes de cutia ao nascer e ao desmame, respectivamente, são, para ambos os sexos, em torno de 145 g e 500 g, 20 e 30 cm e 12 e 17 cm; o ganho de peso médio diário do nascer ao desmame é de $7,76 \mathrm{~g}$.
\end{abstract}

Palavras-chave: comprimento corporal, cutia, perímetro torácico, peso ao nascer

\section{Performance of Agouti (Dasyprocta prymnolopha) Bred in Captivity according to Sex and Parturition in Teresina, Piauí}

\begin{abstract}
This experiment was carried out to evaluate the effect of sex and parturition type (simple or double) on live weight, body length, thoracic diameter at birth and at weaning of agouti, as well as total weight gain and daily mean weight gain, to obtain subsidies that contribute to preservation and rational exploration of these animals. Fourty-one animals (26 males and 15 females) were allotted to a completely randomized a design. Animal weight, thoracic diameter and body length of agouti at birth, for males and females, were respectively: $147.62 \mathrm{~g}$ and $146.25 \mathrm{~g} ; 12.26 \mathrm{~cm}$ and $12.17 \mathrm{~cm} ; 19.91 \mathrm{~cm}$ and $19.39 \mathrm{~cm}$. For the same variables, the values at wean for males and females were, respectively: $481.92 \mathrm{~g}$ and $509.33 \mathrm{~g} ; 17.12 \mathrm{~cm}$ and $16.85 \mathrm{~cm}$; $30.07 \mathrm{~cm}$ and $30.42 \mathrm{~cm}$. No significant difference among the studied variables was observed, when compared to the sex and parturition type (simple or double). It was concluded that the variables studied of of agouti at birth and at weaning were affected neither by sex and nor by parturition type; animal weight, body length and thoracic diameter of agouti at birth and weaning were around $145 \mathrm{~g}$ and $500 \mathrm{~g}, 20$ and $30 \mathrm{~cm}$ and 12 and $17 \mathrm{~cm}$, respectively; and daily mean weight gain from to birth to weaning is about $7.76 \mathrm{~g}$ for both sexes.
\end{abstract}

Key Words: physical length, agouti, thoracic diameter, weight

\section{Introdução}

O interesse mundial pela exploração de novas espécies elevou-se nas últimas décadas e, graças a esse interesse, no Brasil, a legislação e as pesquisas avançam para atender a nova demanda.

A criação comercial de animais silvestres é indicada como alternativa para diversificação de produção e de renda no sul e sudeste da Bahia e em outras regiões do nordeste brasileiro, onde a mão-deobra é pouco onerosa e persistem as dificuldades para implantação da pecuária tradicional (Nogueira Filho \& Nogueira, 2000).

A fauna silvestre representa importante fonte protéica para a alimentação humana, além de proporcionar produtos como pele, couro e pêlos, apresentando grande potencial de mercado e podendo resultar em vantagens econômicas e sociais. Entretanto, exis-

\footnotetext{
1 Professor do Departamento de Zootecnia-CCA/UFPI. E.mail: Iopesjb@uol.com.br

2 Mestrandos em Ciência Animal, Universidade Federal do Piauí. E.mail: renat16@yahoo.com.br

3 Professora do Departamento de Morfofisiologia Veterinária-CCA/UFPI. E.mail: carvalhomam@uol.com.br
} 
tem poucos dados sobre métodos adequados de manejo produtivo e reprodutivo de animais silvestres que poderiam refletir em maior produtividade, viabilizando a criação racional de algumas espécies, com a diminuição da caça predatória das mesmas.

Entre as espécies mais predadas, a cutia (Dasyprocta sp.) tem se destacado por ter sofrido significativa redução de suas populações, tanto pela caça predatória como pela destruição de seu habitat natural (Hosken \& Silveira, 2001). Sua distribuição geográfica vai desde o sul do México, passando pela América Central, até a Argentina, Uruguai, Paraguai e todo território brasileiro (Deutsch \& Puglia, 1988).

Esta pesquisa foi conduzida com o objetivo de avaliar a influência do sexo (macho e fêmea) e do tipo de parto (simples ou duplo) sobre o desempenho de cutias do nascer ao desmame.

\section{Material e Métodos}

A pesquisa foi realizada no Núcleo de Estudos e Preservação de Animais Silvestres do Centro de Ciências Agrárias da Universidade Federal do Piauí, no período de janeiro de 1999 a novembro de 2002, utilizando-se 41 filhotes de cutias (Dasyprocta prymnolopha, Wagler, 1832), sendo 26 machos e 15 fêmeas. O acompanhamento zootécnico se deu no período de janeiro de 1999 a novembro de 2002 na cidade de Teresina-Piauí (latitude $05^{\circ} 05^{\prime} 21^{\prime \prime}$ sul; longitude $42^{\circ} 48^{\prime} 07^{\prime \prime}$ oeste; altitude $72 \mathrm{~m}$; radiação solar mensal $676{\mathrm{cal} . \mathrm{cm}^{2}}^{2}$.dia ${ }^{-1}$; temperatura média $27,3^{\circ} \mathrm{C}$; umidade relativa do ar $70 \%$ (INMET, 1992). Os animais foram mantidos em cativeiro em baias de alvenaria com piso cimentado e tela de alambrado, com boxes dispostos ao longo de um corredor.

Os animais gestantes foram separados em boxe/ maternidade, por ocasião da detecção da gestação, realizada por observação visual e palpação abdominal. Após o parto, os filhotes foram pesados e mensurados semanalmente até os 45 dias, quando ocorreu a desmama. As matrizes foram alimentadas uma vez ao dia com dieta à base de grãos de milho, plantas nativas - bredo (Amaranthus spinosus Linn.) e beldroega (Portulaca oleracea) - verduras, frutas e legumes (abóbora, pepino, cenoura, batata, alface, repolho, melão, melancia, caju e manga) provenientes do restaurante universitário local, além de coco babaçu inteiro e água à vontade.

Para obtenção dos pesos ao nascer e ao desmame, foi utilizada balança digital com precisão de 5,0 g, e, para a medição do perímetro torácico e do comprimento corporal, foi utilizada fita métrica. O ponto de referência para o perímetro torácico foi a cartilagem xifóide do esterno, e a medição do comprimento corporal foi realizada do focinho à base da cauda, passando sobre a cabeça e o dorso do animal

O delineamento experimental adotado foi o inteiramente casualizado, em esquema fatorial $2 \times 2$, sendo associado os tratamentos: sexo (macho, fêmea) e tipo de parto (duplo e simples). A unidade experimental foi representada por um animal. Realizou-se análise de variância e, para comparação das médias, aplicouse o teste SNK (SAS, 1986).

\section{Resultados e Discussão}

Constatou-se que não houve interação entre sexo e tipo de parto para todas as variáveis estudadas $(\mathrm{P}>0,05)$ (Tabela 1).

O peso médio ao nascer, com valores de 147,62 g e 146,25 g para machos e fêmeas, respectivamente, não diferiu entre os sexos $(\mathrm{P}>0,05)$. Os valores obtidos divergiram dos 149,23g encontrados por Medeiros \& Sousa (1996) para ambos os sexos em Dasyprocta prymnolopha. Foram, também, inferiores aos 171,74 $\pm 3,10$ g de Dasyprocta prymnolopha e Dasyprocta aguti, verificados por Pinheiro et al. (1989). Acredita-se que estas diferenças, provavelmente, decorreram do tipo de alimentação desses animais. De acordo com Merrit Júnior (1987), em estudos realizados com Dasyprocta punctata, o peso ao nascer foi de $258 \mathrm{~g}$, enquanto Guimarães (1993), fornecendo, para matrizes Dasyprocta prymnolopha, dieta à base de soja, milho, macaxeira (Manihot utilissima), abóbora (Cucurbita pebo) e ração comercial para aves, registrou peso médio ao nascer de 188,92 g.

Quanto ao tipo de parto, não houve diferença significativa entre o simples e o duplo $(\mathrm{P}>0,05)$. Observou-se, no entanto, que animais provenientes de partos simples apresentaram maior peso ao nascer. Com relação à freqüência de partos, 70,59\% foram duplos e $29,41 \%$ partos simples, o que diverge dos achados de Oliveira et al (2001), ao constatarem $61,7 \%$ de partos duplos.

O peso médio ao desmame de cutias (Dasyprocta prymnolopha) foi de 481,92 g para machos, e de 509,16 g para fêmeas, não diferindo entre si $(\mathrm{P}>0,05)$. Segundo Pinheiro et al. (1989), esses valores para o desmame no período de 35 e 50 dias, respectivamente, 
Tabela 1 - Médias de peso ao nascer e ao desmame ( $\mathrm{g}$ ) de cutias, em função do sexo e do tipo de parto Table 1 - Mean weight at birth and at weaning (g) of agouti influenced by the sex and parturition type

\begin{tabular}{|c|c|c|c|c|c|c|}
\hline \multirow[t]{2}{*}{$\begin{array}{l}\text { Sexo } \\
\text { Sex }\end{array}$} & \multicolumn{3}{|c|}{$\begin{array}{l}\text { Peso ao nascer } \\
\text { Weight at birth } \\
\text { Tipo de parto } \\
\text { Parturition type }\end{array}$} & \multicolumn{3}{|c|}{$\begin{array}{l}\text { Peso ao desmame } \\
\text { Weaning weight } \\
\text { Tipo de parto } \\
\text { Parturition type }\end{array}$} \\
\hline & $\begin{array}{c}\text { Simples } \\
\text { Simple }\end{array}$ & $\begin{array}{l}\text { Duplo } \\
\text { Double }\end{array}$ & $\begin{array}{c}\text { Médias } \\
\text { Means }\end{array}$ & $\begin{array}{l}\text { Simples } \\
\text { Simple }\end{array}$ & $\begin{array}{l}\text { Duplo } \\
\text { Double }\end{array}$ & $\begin{array}{c}\text { Médias } \\
\text { Means }\end{array}$ \\
\hline $\begin{array}{l}\text { Macho } \\
\text { Males }\end{array}$ & 150,00 & 145,24 & 147,62 & 445,00 & 518,85 & 481,92 \\
\hline $\begin{array}{l}\text { Fêmea } \\
\text { Females }\end{array}$ & 147,50 & 145,00 & 146,25 & 515,00 & 503,33 & 509,16 \\
\hline Médias & 148,75 & 145,12 & & 480,00 & 511,09 & \\
\hline
\end{tabular}

Means

Médias, na coluna e linha, não diferem $(p>0,05)$ entre si pelo teste SNK.

Means, within a column and row, do not differ $(p>.05)$ by SNK test.

em Dasyprocta prymnolopha e Dasyprocta aguti foram de 539,70 g e 648,16 g. Medeiros \& Sousa (1996) verificaram peso médio dos filhotes ao desmame de Dasyprocta prymnolopha de 383,33 g para ambos os sexos, valor inferior ao encontrado neste trabalho. Reis et al. (1996), relacionando o peso com a idade em Dasyprocta prymnolopha, observaram, em filhotes com 30 e 60 dias, 473,8 g e 918,4 g, respectivamente, sugerindo a influência da alimentação (ração para roedores com farinha de milho acrescida de farelo de soja, sal mineral e complemento vitamínico) no ganho de peso desses animais.

Os valores para ganho de peso total médio do nascimento ao desmame de cutias (Dasyprocta prymnolopha) foram de 334,30 g e 362,91 g, para machos e fêmeas, e de 331,25 g e 365,97 g, para partos simples e duplos, respectivamente. Os ganhos médios diários do nascimento ao desmame (Tabela 2) foram de 8,07 e 7,43 g/dia, respectivamente, para fêmeas e machos, e para partos simples e duplo, de 7,38 e $8,13 \mathrm{~g} /$ dia, respectivamente, demostrando que as fêmeas ganharam mais peso que os machos e os animais de partos duplos têm maior velocidade de crescimento que os de partos simples durante 0 período do nascimento ao desmame (45 dias). Os valores obtidos na presente pesquisa foram inferiores aos encontrados por Pinheiro et al. (1989), ao constatarem, nos períodos de 35 e 50 dias de idade em Dasyprocta prymnolopha e Dasyprocta aguti, pesos de 363,89 g e 479,22 g e ganhos diários desde o nascimento de $10,40 \mathrm{~g}$ e $9,54 \mathrm{~g}$, respectivamente.

Os dados do comprimento corporal ao nascer e ao desmame encontram-se na Tabela 3. Não houve
Tabela 2 - Médias de ganho de peso diário do nascimento ao desmame $(\mathrm{g})$ de cutias em função do sexo e do tipo de parto

Table 2 - Mean the daily mean weight gain at birth and at weaning (g) of agouti influenced by the sex and parturition type

\begin{tabular}{|c|c|c|c|}
\hline \multirow[t]{3}{*}{$\begin{array}{l}\text { Sexo } \\
\text { Sex }\end{array}$} & \multicolumn{3}{|c|}{$\begin{array}{l}\text { Ganho de peso diário (g) } \\
\text { do nascimento ao desmame } \\
\text { Daily weight gain }(\mathrm{g}) \\
\text { from birth to weaning }\end{array}$} \\
\hline & \multicolumn{3}{|c|}{$\begin{array}{c}\text { Tipo de parto } \\
\text { Birth kind }\end{array}$} \\
\hline & $\begin{array}{l}\text { Simples } \\
\text { Simple }\end{array}$ & $\begin{array}{l}\text { Duplo } \\
\text { Double }\end{array}$ & $\begin{array}{c}\text { Médias } \\
\text { Means }\end{array}$ \\
\hline Macho (Males) & 6,56 & 8,30 & 7,43 \\
\hline Fêmea (Females) & 8,17 & 7,96 & 8,07 \\
\hline Médias (Means) & 7,37 & 8,13 & \\
\hline
\end{tabular}

Médias, na coluna e linha, não diferem $(p>0,05)$ entre si pelo teste SNK.

Means, within a column and row, do not differ ( $p>05)$ by SNK test.

interação entre sexo e tipo de parto para nenhuma das variáveis avaliadas. O comprimento corporal ao nascer não diferiu entre sexo nem entre tipo de parto $(\mathrm{P}>0,05)$. Na literatura referente a este parâmetro não há informações sobre essa variável. Deutsch \& Puglia (1988) citam, no entanto, que o comprimento médio do animal adulto (Dasyprocta aguti) é de 50 $\mathrm{cm}$ do focinho à base da cauda. Em outro trabalho, Lange (1998) destacou que o comprimento total de Dasyprocta azarae foi de $48,3 \mathrm{~cm}$.

Constatou-se que não houve interação entre sexo e tipo de parto para perímetro torácico ao nascer e ao desmame $(\mathrm{P}>0,05)$. Os perímetros torácicos médios 
para machos e fêmeas ao nascer e ao desmame foram, respectivamente, de 12,26 e 12,17 e de 17,16 e 16,85 cm, não havendo diferença entre os tratamentos $(\mathrm{P}>0,05)$. Com relação ao tipo de parto, também não foi observada diferença entre partos simples e duplo, com valores ao nascer e ao desmame, respectivamente de 12,37 e 12,06 e de 17,10 e 16,91 cm (Tabela 4).

Tabela 3 - Médias de comprimento corporal ao nascer e ao desmame (g) de cutias, em função do sexo e do tipo de parto Table 3 - Mean body length at birth and at weaning (g) of agouti influenced by sex and parturition type

\begin{tabular}{|c|c|c|c|c|c|c|}
\hline \multirow[t]{2}{*}{$\begin{array}{l}\text { Sexo } \\
\text { Sex }\end{array}$} & \multicolumn{3}{|c|}{$\begin{array}{l}\text { Comprimento corporal ao nascer } \\
\text { Body length at birth }\end{array}$} & \multicolumn{3}{|c|}{$\begin{array}{c}\text { Comprimento corporal ao desmame } \\
\text { Body length at weaning }\end{array}$} \\
\hline & $\begin{array}{l}\text { Simples } \\
\text { Simple }\end{array}$ & $\begin{array}{l}\text { Duplo } \\
\text { Double }\end{array}$ & $\begin{array}{l}\text { Médias } \\
\text { Means }\end{array}$ & $\begin{array}{l}\text { Simples } \\
\text { Simple }\end{array}$ & $\begin{array}{l}\text { Duplo } \\
\text { Double }\end{array}$ & $\begin{array}{l}\text { Médias } \\
\text { Means }\end{array}$ \\
\hline $\begin{array}{l}\text { Macho } \\
\text { Males }\end{array}$ & 20,40 & 19,43 & 19,91 & 30,30 & 29,84 & 30,07 \\
\hline $\begin{array}{l}\text { Fêmea } \\
\text { Females }\end{array}$ & 19,55 & 19,24 & 19,39 & 30,65 & 30,20 & 30,42 \\
\hline $\begin{array}{l}\text { Médias } \\
\text { Means }\end{array}$ & 19,97 & 19,33 & & 30,47 & 30,02 & \\
\hline
\end{tabular}

Médias, na coluna e linha, não diferem $(P>0,05)$ entre si pelo teste SNK.

Means, within a column and row, do not differ $(p>$.05) by SNK test.

Tabela 4 - Médias de perímetro torácico ao nascer e ao desmame ( $\mathrm{g}$ ) de cutias, em função do sexo e do tipo de parto Table 4 - Average thoracic diameter at birth and at weaning $(\mathrm{g})$ of agouti influenced by the sex and parturition type

\begin{tabular}{|c|c|c|c|c|c|c|}
\hline \multirow[t]{2}{*}{$\begin{array}{l}\text { Sexo } \\
\text { Sex }\end{array}$} & \multicolumn{3}{|c|}{$\begin{array}{l}\text { Perímetro torácico ao nascer } \\
\text { Thoracic perimeter at birth }\end{array}$} & \multicolumn{3}{|c|}{$\begin{array}{l}\text { Perímetro torácico ao desmame } \\
\text { Thoracic perimeter at weaning }\end{array}$} \\
\hline & $\begin{array}{l}\text { Simples } \\
\text { Simple }\end{array}$ & $\begin{array}{l}\text { Duplo } \\
\text { Double }\end{array}$ & $\begin{array}{l}\text { Médias } \\
\text { Means }\end{array}$ & $\begin{array}{l}\text { Simples } \\
\text { Simple }\end{array}$ & $\begin{array}{l}\text { Duplo } \\
\text { Double }\end{array}$ & $\begin{array}{c}\text { Médias } \\
\text { Means }\end{array}$ \\
\hline Macho (Males) & 12,54 & 11,98 & 12,26 & 17,20 & 17,12 & 17,16 \\
\hline Fêmea (Females) & 12,20 & 12,14 & 12,17 & 17,00 & 16,70 & 16,85 \\
\hline Médias (Means) & $12,37^{\mathrm{a}}$ & 12,06 & & 17,10 & 16,91 & \\
\hline
\end{tabular}

Médias, na coluna e linha, não diferem $(P>0,05)$ entre si pelo teste SNK.

Means, within a column and row, do not differ ( $p>05)$ by SNK test. 


\section{Conclusões}

Os pesos ao nascer e ao desmame de filhotes de cutia não são influenciados pelo sexo nem pelo tipo de parto.

Em projetos de criação de cutia em cativeiro nos moldes deste experimento, pode-se estabelecer índices zootécnicos em que o peso médio, o comprimento corporal e o perímetro torácico de filhotes de cutia ao nascer e ao desmame são, para ambos os sexos, em torno de 145 g e $500 \mathrm{~g}$; de 20 e $30 \mathrm{~cm}$; de 12 e $17 \mathrm{~cm}$, respectivamente. $\mathrm{O}$ ganho de peso médio diário do nascer ao desmame é de 7,76 g para ambos os sexos.

\section{Literatura Citada}

DEUTSCH, L.A.; PUGLIA, L.R.R. Os animais silvestres. Rio de Janeiro: Globo, 1988. p.51-60.

GUIMARÃES, D.A.A. Algumas características reprodutivas de cutia fêmea Dasyprocta prymnolopha (Wagler, 1831) criada em cativeiro. Belém: Universidade Federal do Pará, 1993. 89p. Dissertação (Mestrado em Zoologia) - Universidade Federal do Pará, 1993.

HOSKEN, F.M.; SILVEIRA, A.C. Criação de cutias. Viçosa, MG: Aprenda fácil, 2001. v.4, p.21-22.

INMET. Instituto Nacional de Meteorologia: Normas climatológicas do período 1960-1990, Brasília, 1992.

LANGE, R.R. Criação e relocação de cutias Dasyprocta azarae (Lichtenstein, 1823) em área verde urbana, Curitiba-PR. Curitiba: Universidade Federal do Paraná, 1998. 131p. Dissertação (Mestrado em Zoologia) - Universidade Federal do Paraná, 1998.

MEDEIROS, C.P.S.; SOUSA, M.S.N. Ganho de peso do nascer ao desmame de cutia (Dasyprocta prymnolopha), criada em cativeiro. In: CONGRESSO PANAMERICANO DE CIÊNCIAS VETERINÁRIAS, 15., 1996, Campo Grande. Abstracts... Campo Grande: Associação Panamericana de Ciências Veterinárias, 1996. p.78.
MERITT JR,. D.A. The ecology of the central american agouti, Dasyprocta punctata. Chicago: University of Illinois, 1987. 240p. Dissertação (Mestrado em Zoologia) - University of Illinois, 1987.

NOGUEIRA FILHO, S.L.G.; NOGUEIRA, S.S.C. Criação comercial de animais silvestres: produção e comercialização da carne e de subprodutos na região sudeste da Brasil. Revista Econômica do Nordeste, v.31, p.188-195, 2000.

OLIVEIRA, M.F.; MACEDO, C.R.; FEIJÓ, F.M.C. et al. Dados do manejo reprodutivo de cutias criadas em cativeiro (Dasyprocta aguti). In: CONGRESSO DA ASSOCIAÇÃO BRASILEIRA DE VETERINÁRIOS DE ANIMAIS SELVAGENS, 5., 2001, São Paulo. Anais... São Paulo: Associação Brasileira de Veterinários de Animais Silvestres, 2001. p.68.

PINHEIRO, M.J.P.; ANDRADE, S.A.; CUNHA, J.N. Preservação e exploração de animais silvestres: preá, cutia e mocó. Caatinga, v.6, p.28-49, 1989.

REIS, A.N.; PEREIRA, M.A.P ; SILVA, A.O.A. et al. Avaliação de alguns parâmetros de desenvolvimento em cutias (Dasyprocta prymnolopha) criada em cativeiro. In: CONGRESSO PANAMERICANO DE CIÊNCIAS VETERINÁRIAS, 15., 1996, Campo Grande. Abstracts... Campo Grande: Associação Panamericana de Ciências Veterinárias, 1996. p.69.

STATISTICAL ANALYSIS SYSTEM - SAS. SAS System for linear models. Cary: 1986. 211p.

Recebido em: 30/04/03

Aceito em: 28/05/04 